\title{
Leptomeningeal Metastasis from Carcinoma Breast: A Case of Unknown to Known
}

\author{
Princy S. Soman, Hemalatha A*, Kalyani R and Lakshmaiah V \\ Sri Devaraj Urs Academy of Higher Education and Research centre (Pathology and Sri Devaraj Urs Medical College)
}

\begin{abstract}
Leptomeningeal metastasis is known to occur as an initial presentation in solid tumors. The presentation of meningeal symptoms before the diagnosis of primary tumor is seen in $10-20 \%$ cases. Though the sophisticated radiological imaging is available, Cerebrospinal Fluid (CSF) analysis for tumor cells remains the gold standard method for diagnosis. Incidence of brain metastasis from primary carcinoma breast is about $3-8 \%$ only.

We present a case of young female who presented with neurological symptoms and misdiagnosed as meningitis on Magnetic Resonance Imaging (MRI), but later after CSF analysis it was diagnosed as metastases from carcinoma breast.
\end{abstract}

Keywords: Leptomeningeal Metastasis, Carcinoma Breast, Meningitis

\section{Introduction}

Leptomeningeal metastasis is involvement of Cerebrospinal Fluid (CSF) and leptomeninges by tumor cells. The diagnosis of leptomeningeal metastasis depends on clinical features, imaging and detection of malignant cells in CSF. ${ }^{[1]}$ Malignancies from lungs, breast, malignant melanoma, leukemia, lymphoma have known to have propensity towards meninges. ${ }^{[2]}$ The presentation with meningeal symptoms before the diagnosis of primary solid tumor is seen in $10-20 \%$ of cases. ${ }^{[3]}$ About $15 \%$ of patients with brain metastases have no clearly detected primary tumor despite being thoroughly evaluated. ${ }^{[4]}$ But the diagnosis of such presentations has increased due to the advanced imaging techniques and screening protocols. ${ }^{[5]}$

Here we present a case of 45-year-old female who presented to emergency department with symptoms of Leptomeningitis and later diagnosed as carcinomatous meningitis due to infiltrating ductal carcinoma of breast.

\section{Case Report}

A 45-year-old comatose female presented to Emergency Medicine Department of our tertiary hospital from a private hospital because of deterioration of her general condition. Patient attender gave history of headache and vomiting for 10 days, altered sensorium for 3 days. Headache was throbbing in nature. Vomiting was non-bloody, non-bilious and projectile in nature. Patient attender also gave history of photophobia. She was known hypertensive on irregular medication. There was no history of fever or any other co morbidities. Neurological examination showed signs of increased intra cranial tension with poor Glasgow coma scale (6/15). Complete blood count revealed Microcytic hypochromic anemia with neutrophilic leucocytosis. Magnetic Resonance Imaging done outside showed leptomeningeal enhancement without parenchyma lesion favouring meningoencephalitis. Lumbar puncture was done under aseptic precautions and CSF was collected. CSF biochemical analysis was normal except reduction in proteins $(5 \mathrm{mg} / \mathrm{dl})$. No growth was seen in culture.

CSF was clear in appearance and cell count was 250cells/ $\mathrm{cu} \mathrm{mm}$. CSF was centrifuged and then smears were examined from the centrifuged deposit of the fluid showing high cell yield. (Fig 1). Smear showed cells arranged in dispersed pattern which were round to polygonal in shape with increased nuclear cytoplasmic ratio $(\mathrm{N}: \mathrm{C}$ ratio), nucleus showing hyperchromasia and chromatin was coarse granular irregularly distributed. Few of the cells showed irregular nuclear membrane with moderate amount of eosinophilic cytoplasm. A few abnormal mitotic figures were also seen. Background was clear. Impression was given as: Smear positive for malignancy suggesting adenocarcinoma metastatic deposits.

In view of patient being female left breast examination revealed a lump measured $3 \times 2 \mathrm{cms}$ in the central compartment. It was firm to hard in consistency and attached to nipple areolar complex. It was not associated with nipple discharge. Also left axillary lymph node was measuring $2 \times 1 \mathrm{cms}$ and left supra clavicular lymph node was palpable measuring $1 \mathrm{x} 1 \mathrm{~cm}$. After getting consent from patient attender FNAC from the breast lump was done. 
Smears from the left breast lump aspirate (Fig 2) showed satisfactory cell yield consisting of ductal epithelial cells arranged in loose cohesive clusters, sheets and in singles. Cells showed hyperchromatic nucleus, with increased N:C ratio and irregularity of the nuclear borders. These cells showed marked pleomorphism. These tumour cells were also seen adhering to adipocytes Myoepithelial cells not seen in the smear studied. Background showed fibrocollagenous tissue and erythrocytes.

FINAL IMPRESSION was given as: Features Consistent with malignant lesion suggestive of Infiltrating ductal carcinoma - Left breast. FNAC of left supraclavicular lymph node showed tumour deposits.

Trucut biopsy was done for Hormonal studies showed ER, PR, Her2 neu to be negative suggesting triple negative cancer. (Fig 3: Immunohistochemistry of ER, PR, Her2neu and Ki-67). Final diagnosis was given as Carcinomatous meningitis secondary to Infiltrating ductal carcinoma of left breast - Stage IV.

\section{Discussion}

Leptomeningeal metastasis is caused by malignant cells which infiltrate the CSF by hematogenous spread, endo or peri neural dissemination along peripheral nerves or by direct expansion of parenchymal cerebral metastases. Leptomeningeal metastases usually present with widely metastatic and progressive cancer $(70 \%)$ but it can occasionally be the first manifestation in $5-10 \%$ cases. ${ }^{[6]}$

Leptomeningeal metastasis from carcinoma breast has been known to occur $3-8 \%$ of cases. ${ }^{[7]} \mathrm{CSF}$ examination is considered as gold standard in examination of leptomeningeal metastasis. ${ }^{[8]}$ The risk factors for brain metastases include young age, tumor stage, human epidermal growth factor receptor 2 positivity, triple negativity, number of metastases $(n>2)$ and large tumor size. Median duration for brain metastases is about 2-3 years after the initial diagnosis of breast cancer. The overall survival of patients with brain metastases is extremely poor and these metastases are less responsive to systemic therapies. ${ }^{[9]}$

A triad of clinical signs, CSF analysis, Imaging techniques helps in early diagnosis of CNS involvement in primary as well as secondary tumor. CSF cytological study has a high specificity. ${ }^{[10]}$ Morphological detection of tumor cells in CSF and the presence of tumor markers in CSF can be used for diagnosis, staging and deciding appropriate therapy. CSF flow cytometry is useful in detecting CNS involvement in high-risk individuals. ${ }^{[1]}$

Ancillary studies in CSF like tumor markers, flowcytometry and PCR can help in increasing diagnostic yield especially in cases of Carcinoma of Unknown Primary (CUP). ${ }^{[12]}$ Tumor markers in CSF such as CEA, AFP, $\beta-$ HCG and monoclonal immunoglobulins has varying sensitivity and specificity. Specific tumor markers identified in the CSF, especially if higher than in serum in the appropriate clinical context can assist in the diagnosis. Clinical judgement will be essential to guide and establish final diagnosis. Flow cytometry is considered more sensitive than cytology for detection of hematologic malignant cells in the CSF not applicable in solid tumors. Earlier studies reported that

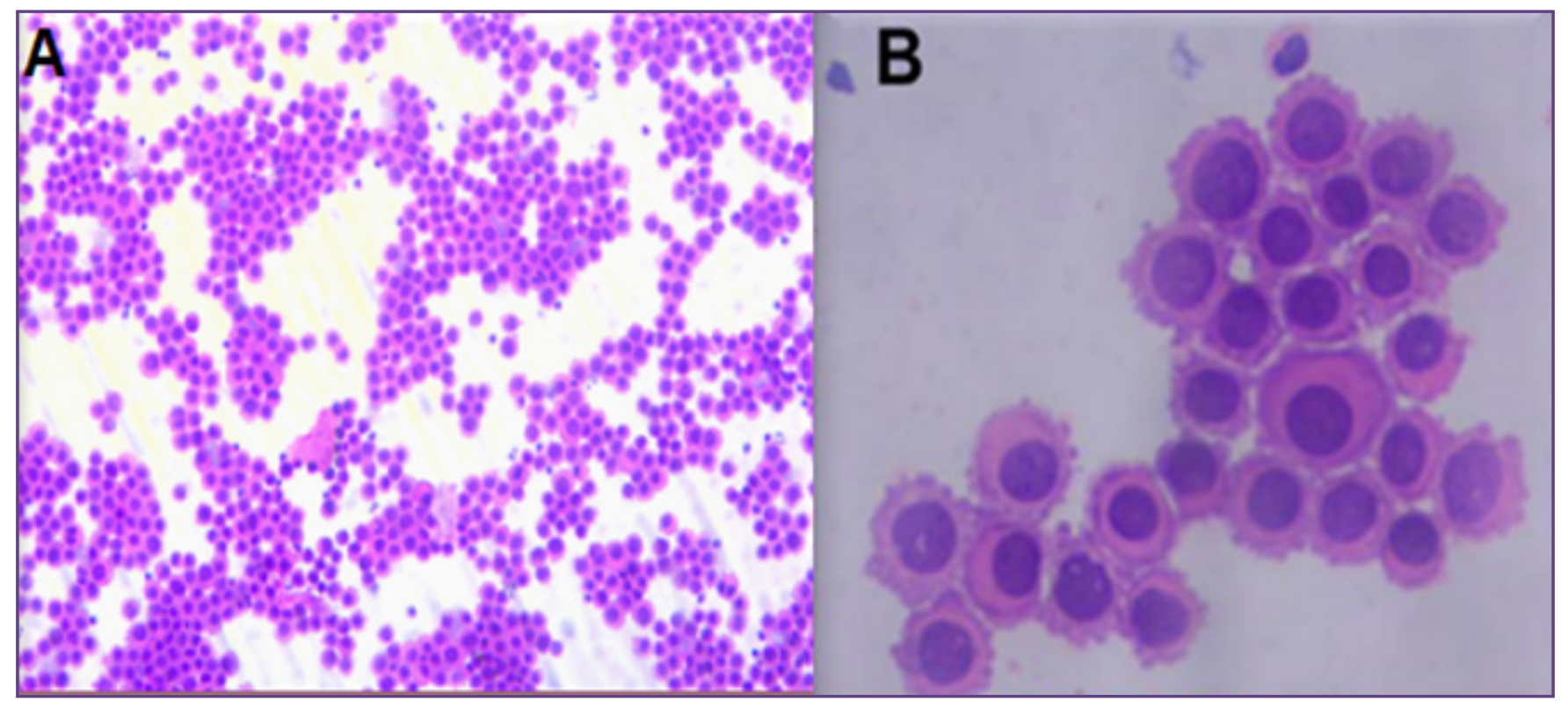

Fig. 1: centrifuged deposit of the fluid showing high cell yield. 


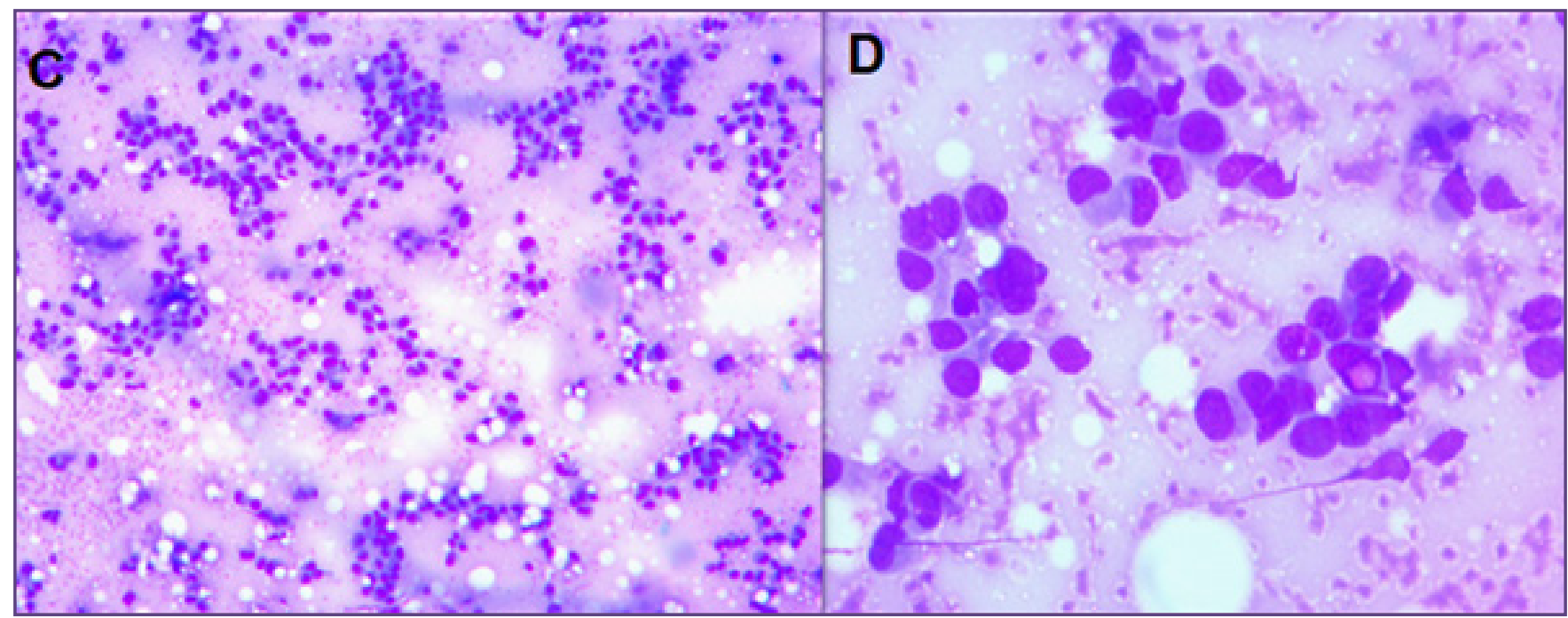

Fig. 2: Smears from the left breast lump aspirate.

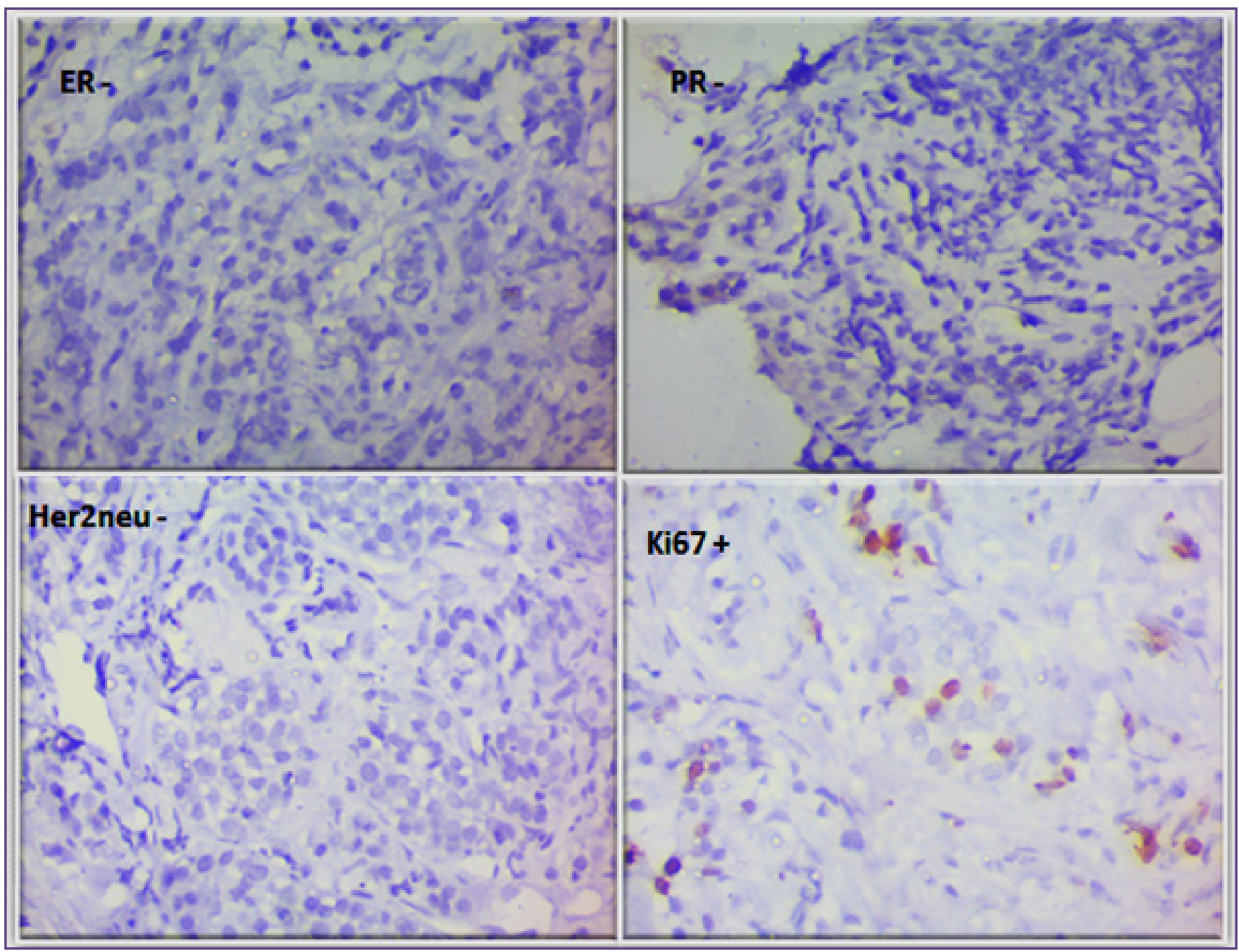

Fig. 3: Immunohistochemistry of ER, PR, Her2neu and Ki-67. 
elevated levels of VEGF in CSF were sensitive and highly specific for the diagnosis of leptomeningeal metastasis from breast cancer, lung cancer and melanoma. ${ }^{[10]}$

According to RANO group CSF cytology and high-quality gadolinium enhanced MRI is helpful in making diagnosis of metastasis. ${ }^{[12]}$ However in our case MRI was not helpful for the diagnosis. MRI showing leptomeningeal enhancement without parenchymal lesion favouring metastasis from adenocarcinoma.

In breast carcinoma Metastasis is seen in lobular histologic type and triple negative subtype and is usually associated with short median survival of few months in spite of aggressive treatment. Our patient presented to Emergency Medicine Department (EMD) with clinical feature of leptomeningitis without any previous history of malignancy. Similar findings have been described in a study where in one case of lung carcinoma, one case of gastric carcinoma and another case of CUP which presented to EMD without any history of known malignancy. ${ }^{[8]}$ Even though combination of treatment with surgery, radiation and intrathecal chemotherapy has been suggested but the prognosis is poor in these patients.

\section{Conclusions}

Patients with carcinomabreastcan present as leptomeningeal metastasis of unknown primary. A high clinical suspicious and CSF analysis may help in accurate diagnosis of such cases and hence even in spite of availability of extensive screening protocols and diagnostic modalities for early detection of breast carcinomas, patients can still present with symptoms of metastasis due to social stigma attached with the disease.

\section{References}

1. Pan Z, Yang G, He H,Yuan T, Wang Y, Li Y et al. Leptomeningeal metastasis from solid tumors: clinical features and its diagnostic implication. Sci. Rep 2018; 8:1-13.
2. Witzel I, Ferrer L O, Pantel K, Muller V and Wikman H. Breast cancer brain metastases: biology and new clinical perspectives. Breast Cancer Research 2016; 18:8 1-9

3. Niwinska A, Rudnicka H, Murawska M. Breast cancer leptomeningeal metastasis: propensity of breast cancer subtypes for leptomeninges and the analysis of factors influencing survival. Med Oncol 2013;30: 408.

4. Polyzoidis KS, Miliaras G, Pavlidis N. Brain metastasis of unknown primary: a diagnostic and therapeutic dilemma. Cancer Treat Rev 2005; 31: 247-55.

5. Niwinska A, Murawska M, Pogoda K. Breast cancer brain metastases: differences in survival depending on biological subtype, RPA RTOG prognostic class and systemic treatment after whole-brain radiotherapy (WBRT). Ann. Oncol 2010; 21:942-8.

6. Rhun EL, Preusser M, Bent MV, Andratschke N, Weller $\mathrm{M}$. How we treat patients with leptomeningeal metastases. ESMO open 2019; 4:507-9.

7. Li X, Zhang Y, Ding J, Wang M, Li N, Yang H et al. Clinical significance of detecting CSF-derived tumor cells in breast cancer patients with leptomeningeal metastasis. Oncotarget 2018; 9:2705-14.

8. Ahn S, Lim K S. Three Cases of Neoplastic Meningitis Initially Diagnosed with Infectious Meningitis in emergency department. Case Rep Emerg Med 2013; 10:1-4.

9. Cheng X, Hung MC. Breast cancer brain metastases. Cancer Metastasis Rec 2007; 26: 635-43.

10. Leal T, Chang JE, Mehta M, Robins HI. Leptomeningeal Metastasis: Challenges in Diagnosis and Treatment. Curr Cancer Ther Rev 2011; 7: 319-27.

11. Ning M, Chunhua M, Rong J, Yuvan L, Jinduo J, Bin W et al. Diagnostic value of circulating tumor cells in cerebrospinal fluid. Open Med 2016;11: 21-24.

12. Nayar G, Ejikema T, Chongsathidkiet P, Elsamadicy AA, Blackwell K L, Clarke J M et al. Leptomeningeal disease: current diagnostic and therapeutic strategies. Oncotarget 2017; 8: 73312-28.

\footnotetext{
*Corresponding author:

Dr. Hemalatha A, Sri Devaraj Urs Medical College

Phone: +91 9972212324

Email: drhemashashi@gmail.com
}

Financial or other Competing Interests: None.
Date of Submission : 17/01/2020

Date of Final Revision : 18/02/2021

Date of Acceptance : 22/02/2021

Date of Publication : 30/03/2021 